\title{
Red Thermally Activated Delayed Fluorescence in Dibenzopyridoquinoxaline-Based Nanoaggregates
}

\author{
Subhadeep Das ${ }^{\text {(i) }}$ \\ Subhankar Kundua ${ }^{\text {(iD }}$ \\ Bahadur Sk ${ }^{\text {a }}$ \\ Madhurima Sarkara ${ }^{(\mathbb{D})}$ \\ Abhijit Patra*a \\ a Department of Chemistry, Indian Institute of Science Education \\ and Research Bhopal, Bhauri, Bhopal, Madhya Pradesh, 462066, \\ India. \\ *abhijit@iiserb.ac.in
}

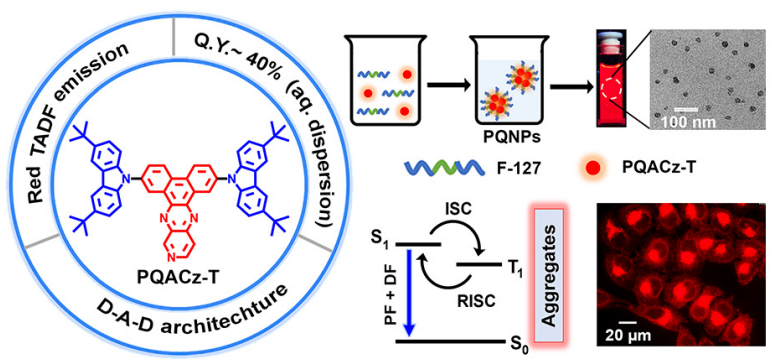

\section{Introduction}

Received: 01.08.2021

Accepted after revision: 26. 10.2021

DOI: 10.1055/a-1679-9558; Art ID: OM-2021-08-0038-OA

\section{License terms: $@(1) \circledast$}

(c) 2021. The Author(s). This is an open access article published by Thieme under the terms of the Creative Commons Attribution-NonDerivative-NonCommercial License, permitting copying and reproduction so long as the original work is given appropriate credit. Contents may not be used for commercial purposes, or adapted, remixed, transformed or built upon. (https://creativecommons.org/licenses/by-nc-nd/4.0/)

Abstract All-organic thermally activated delayed fluorescence (TADF) materials have emerged as potential candidates for optoelectronic devices and biomedical applications. However, the development of organic TADF probes with strong emission in the longer wavelength region $(>600 \mathrm{~nm}$ ) remains a challenge. Strong $\pi$-conjugated rigid acceptor cores substituted with multiple donor units can be a viable design strategy to obtain red TADF probes. Herein, 3,6-di-t-butyl carbazole substituted to a dibenzopyridoquinoxaline acceptor core resulted in a Tshaped donor-acceptor-donor compound, PQACZ-T, exhibiting red TADF in polymer-embedded thin-films. Further, PQACz-T self-assembled to molecular nanoaggregates of diverse size and shape in THF-water mixture showing bright red emission along with delayed fluorescence even in an aqueous environment. The self-assembly and the excitedstate properties of PQACz-T were compared with the nonalkylated analogue, PQCz-T. The delayed fluorescence in nanoaggregates was attributed to the high rate of reverse intersystem crossing. Moreover, an aqueous dispersion of the smaller-sized, homogeneous distribution of fluorescent nanoparticles was fabricated upon encapsulating PQACZ-T in a triblock copolymer, F-127. Cytocompatible polymer-encapsulated PQACZ-T nanoparticles with large Stokes shift and excellent photostability were demonstrated for the specific imaging of lipid droplets in HeLa cells.

Key words: TADF, dibenzopyridoquinoxaline, nanoaggregates, fluorescence imaging, lipid droplets
All-organic thermally activated delayed fluorescence (TADF) materials have drawn interest recently due to their potential in high-performance organic light-emitting diodes with nearly $100 \%$ theoretical internal efficiency. ${ }^{1-6}$ Molecules with a small energy gap $\left(\Delta E_{\mathrm{ST}}\right)$ between the lowest energy singlet $\left(S_{1}\right)$ and triplet states $\left(T_{1}\right)$ favour an efficient reverse intersystem crossing (RISC) process. ${ }^{1,4-9} A$ small $\Delta E_{\mathrm{ST}}$ can be obtained by minimizing the overlap between the frontier molecular orbitals, i.e., HOMO and LUMO. ${ }^{4-12}$ In this context, various design strategies, including donor-acceptor (D-A) or donor- $\pi$-acceptor $(D-\pi-A)$ architecture, and the introduction of multiple strong donors or acceptor units with twisted molecular structures resulting in significant intramolecular charge transfer (ICT), have been explored. ${ }^{4,10,12-20}$ However, as low orbital overlap results in weaker oscillator strength $(f)$, achieving simultaneously high photoluminescence (PL) quantum yield and small $\Delta E_{\mathrm{ST}}$ is challenging. ${ }^{3}$ Fine-tuning the orbital overlap by the introduction of rigid or flexible $\pi$-spacers has often been used to overcome this issue. ${ }^{7,21,22}$

Recently, efficient blue and green TADF emitters have been developed employing the twisted $D-\pi-A$ architecture. ${ }^{22,23}$ However, optimizing the design strategy to obtain highly efficient red-emitting TADF materials suitable for organic photovoltaics, sensors, or intracellular imaging still remains elusive. ${ }^{24-28}$ The dominance of nonradiative deactivations at the higher wavelength according to the energy gap law along with the low oscillator strength due to weak D-A conjugation limits the efficiency of red TADF emitters. ${ }^{29}$ Thus, strong $\pi$-conjugated rigid acceptor cores are desired to provide deep LUMO levels resulting in low bandgap energy along with suppressed vibrational motions. However, 
strong $\pi-\pi$ stacking interactions may also quench the fluorescence in the aggregated or solid-state. ${ }^{30,31}$

The quenching of emission can be circumvented by inducing twisted molecular structures or by introducing bulky terminal substituents into the donor/acceptors, thus reducing the $\pi-\pi$ stacking in the aggregated state. ${ }^{32-36}$ Herein, we developed a T-shaped molecular TADF emitter, PQACz-T, using dibenzo[f,h]pyrido[3,4-b]quinoxaline (DBPQ) as a rigid acceptor and 3,6-di-t-butyl carbazole (DTCz) as a donor. We anticipated that the introduction of bulky tert-butyl groups at the 3,6-positions of carbazole would increase the donor strength and might reduce the $\pi-\pi$ stacking in the aggregated state. Comparing with the nonalkylated analogue, PQACZ-T exhibited enhanced red TADF emission in the aqueous dispersion of nanoaggregates and thin films. Steadystate and time-resolved emission measurements coupled with computational investigations were carried out to elucidate the TADF properties. Furthermore, the amphiphilic triblock copolymer-encapsulated PQACz-T nanoparticles with an average diameter of $20 \pm 4 \mathrm{~nm}$ were employed for the intracellular imaging of lipid droplets (LDs).

\section{Results and Discussion}

The synthetic route of PQACz-T is illustrated in Scheme 1 Friedel-Crafts alkylation of carbazole using $t$-butyl chloride resulted in the donor unit, DTCz (Scheme S1). 2,7-Dibromophenanthrene-9,10-dione (P2) was synthesized via the bromination of phenanthrene-9,10-dione (P1) using $N$-bromosuccinimide under acidic conditions. The key intermediate compound, 2,7-dibromodibenzo[f,h]pyrido[3,4- $b]$ quinoxaline (P3), was synthesized by the Schiff base condensation between P2 and 3,4-diaminopyridine. Subsequently, the Pd-catalysed Buchwald-Hartwig coupling between P3 and $\mathrm{DTC} z$ led to the desired product PQACz-T with approximately $65 \%$ yield. The nonalkylated analogue, PQCz-T, was synthesized following the protocol developed by our group earlier (Scheme 1). ${ }^{37}$ The purity of PQACz-T and all the intermediate compounds was ascertained by ${ }^{1} \mathrm{H}-\mathrm{NMR},{ }^{13} \mathrm{C}-\mathrm{NMR}$ spectroscopy, and high-resolution mass spectrometry [Supporting Information (SI) Figures S37-S46].

PQACZ-T showed multiple absorption peaks in solvents of varying polarity (Figure 1a, SI, Figure S1, Table S1). The intense absorption bands at $\sim 359 \mathrm{~nm}\left(\varepsilon \sim 2.4 \times 10^{4} \cdot \mathrm{M}^{-1} \cdot \mathrm{cm}^{-1}\right)$ and $\sim 390 \mathrm{~nm}\left(\varepsilon \sim 4.9 \times 10^{3} \cdot \mathrm{M}^{-1} \cdot \mathrm{cm}^{-1}\right)$ were attributed to the $\pi-\pi^{*}$ transitions of the donor (DTCz) and acceptor (DBPQ) units, respectively (Figure $1 \mathrm{a}$, SI, Figure $\mathrm{S} 1$ ). The red-shifted broad and weak absorption band $\left(\varepsilon \sim 1.2 \times 10^{3} \cdot \mathrm{M}^{-1} \cdot \mathrm{cm}^{-1}\right)$ at $\sim 400-550 \mathrm{~nm}$ might be due to the ICT from the donors (DTCz) to the acceptor (DBPQ) unit. ${ }^{18}$ The optical bandgap of PQACz-T was estimated from the onset of absorption, and it was found to be $2.35 \mathrm{eV}$ (SI, Figure S2).

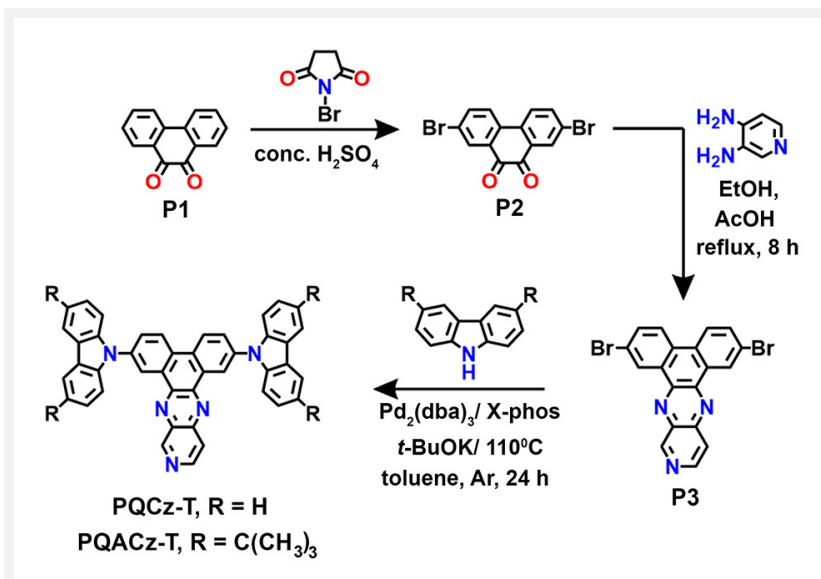

Scheme 1 The synthetic scheme of dibenzopyridoquinoxaline-based T-shaped D-A-D molecules PQCZ-T and PQACZ-T.

The emission spectra of PQACZ-T in solvents of varying polarity revealed a bathochromic shift in emission maxima from $538 \mathrm{~nm}$ (hexane; green) to $697 \mathrm{~nm}$ (dichloromethane; red, Figure 1b). The full-width at half-maximum (FWHM) also gradually increased from $88 \mathrm{~nm}$ (hexane) to $158 \mathrm{~nm}$ (dichloromethane). The large positive solvatochromic shift, along with the gradual increase in the FWHM, suggested a typical excited-state ICT character (Figure S4). ${ }^{18,37-39}$ The fluorescence quantum yield in toluene was found to be $\sim 37 \%$, which decreased gradually with increasing solvent polarity $(<1 \%$ in dichloromethane, SI, Table S2). The red shift and decrease in quantum yields in polar solvents were associated with the enhanced nonradiative transitions and could be due to the twisted ICT (TICT). ${ }^{37,40,41}$ The basic spectroscopic properties and the charge transfer characteristics of PQCz-T were reported in our previous study (SI, Figure S5, Table S3). ${ }^{37}$ The emission maxima of PQACz-T $\left(\lambda_{\mathrm{em}}=590 \mathrm{~nm}\right.$, toluene) showed a bathochromic shift as compared to PQCz-T $\left(\lambda_{\mathrm{em}}=564 \mathrm{~nm}\right.$, toluene) due to the presence of electron-donating alkyl units in the donor DTCz.

The transient PL decay measurements exhibited bi-exponential decay for PQACZ-T, which could be due to the existence of locally excited (LE) and charge transfer (CT) states (SI, Figure S6, Table S4). ${ }^{18}$ The reconstructed emission spectra of PQACz-T obtained through the time resolved emission spectra (TRES) analysis at $1.1 \mathrm{~ns}$ showed a distinct spectral profile resembling the emission spectrum of DTC $z$ in toluene, referring to the LE-state emission (SI, Figure S7). However, the steady-state emission spectrum was significantly red-shifted and originated from the CT state. A broad emission spectrum with a peak maximum at $608 \mathrm{~nm}$ was observed for PQACz-T in toluene at $77 \mathrm{~K}$ (SI, Figure S8). TRES measurements were carried out in toluene at room temperature and $77 \mathrm{~K}$ to obtain the energy difference between the singlet and triplet states (Figure $1 \mathrm{c}$ ). The $\mathrm{S}_{1}$ and $\mathrm{T}_{1}$ energy 
(a)
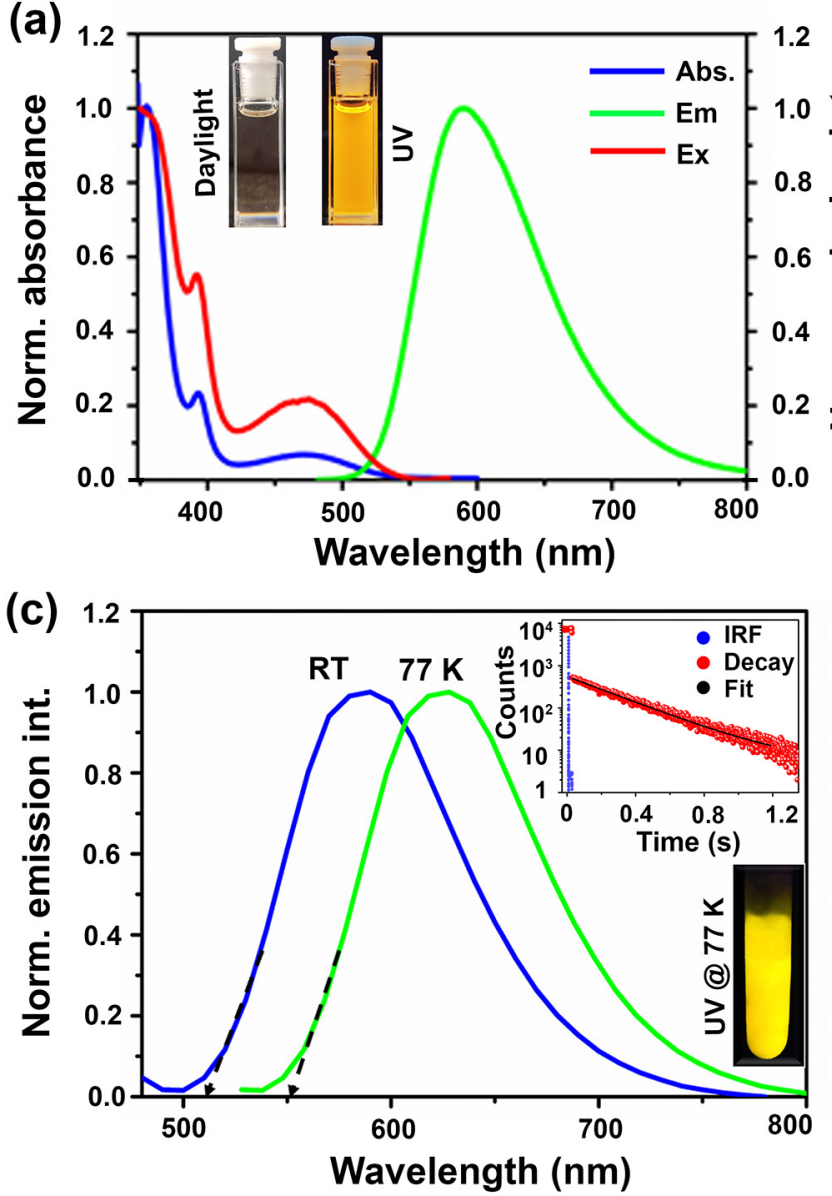

(b)

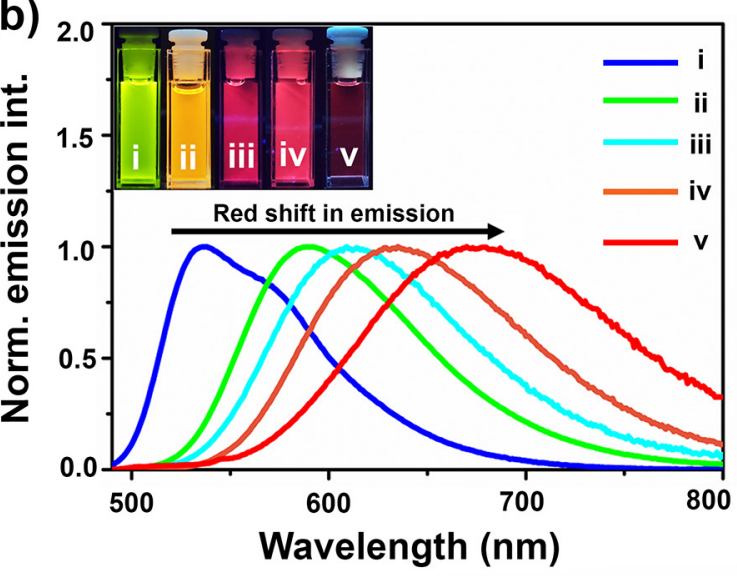

(d)

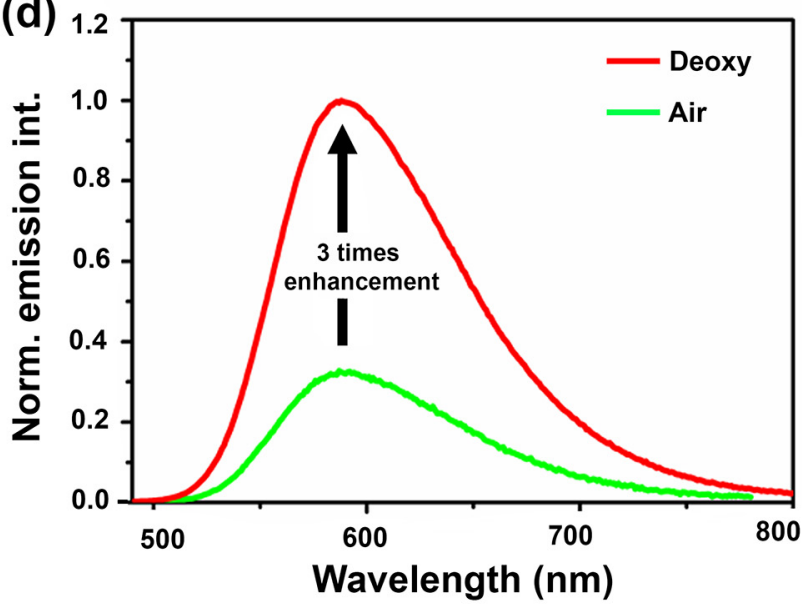

Figure 1 (a) Normalized absorption, emission, and excitation spectra of PQACz-T in toluene under ambient conditions; inset: digital photographs of PQACz-T in toluene under daylight and UV irradiation $\left(\lambda_{\mathrm{ex}}=365 \mathrm{~nm}\right.$ ). (b) Normalized emission spectra of PQACz-T in (i) hexane, (ii) toluene, (iii) dioxane, (iv) tetrahydrofuran, and (v) dichloromethane; inset: digital photographs depicting the solvatochromic shift in the emission spectra with increasing solvent polarity under UV lamp illumination $\left(\lambda_{\mathrm{ex}}=365 \mathrm{~nm}\right.$ ). (c) Time-resolved emission spectra (TRES) of PQACz-T in toluene at room temperature (time delay: $10 \mathrm{~ns}$, blue) and liq. $\mathrm{N}_{2}$ temperature ( $77 \mathrm{~K}$, time delay: $280 \mathrm{~ms}$, green). The inset figures depict the transient PL decay of PQACz-T at $77 \mathrm{~K}$ and the digital photograph showing phosphorescence at $77 \mathrm{~K}$ under UV irradiation. (d) Emission spectra of PQACZ-T in toluene at room temperature under air (green) and after deoxygenation with prolonged $\mathrm{N}_{2}$ purging (red). For (a-d), the concentration of PQACz-T was kept constant at $10 \mu \mathrm{M}, \lambda_{\mathrm{ex}}=470 \mathrm{~nm}$.

levels were estimated from the onset of fluorescence (room temperature measurement, time delay: $10 \mathrm{~ns}$ ) and phosphorescence ( $77 \mathrm{~K}$ measurement, time delay: $280 \mathrm{~ms}$ ) spectra and were found to be $\sim 2.44$ and $2.24 \mathrm{eV}$, respectively, resulting in a $\Delta E_{\mathrm{ST}}$ of $0.20 \mathrm{eV}$ (Figure 1c).

A three-times enhancement in the emission intensity of PQACZ-T in toluene was noticeable upon deoxygenation with prolonged nitrogen-purging, indicating the possibility of fluorescence quenching by molecular oxygen (Figure 1d). ${ }^{42,43}$ Moreover, upon deoxygenation, a long-lived emission was observed in the microsecond time domain $\left(\tau_{\text {avg }}=4.5 \mu \mathrm{s}\right.$, Figure $2 \mathrm{a}$, SI, Table S5). TRES measurements at room temperature revealed that the decay-associated emission spectra at the microsecond (time delay: $4.5 \mu \mathrm{s}$ ) and nanosecond (time delay: $10 \mathrm{~ns}$ ) timescales were identical, indicating the delayed fluorescence phenomenon in PQACZ-T (Figure 2b). The activation energy of RISC for $\mathrm{PQACZ}-\mathrm{T}$ in toluene was found to be $0.14 \pm 0.03 \mathrm{eV}$, very close to the $\Delta E_{S T}$ value (Figure 2c, SI, Table S6). ${ }^{1}$ Furthermore, the laser power-dependent emission study was performed to assign the nature of the delayed fluorescence. ${ }^{7,12,18}$ The linear dependence of the delayed fluorescence intensity as a function of laser power, with a slope of 1.04 , confirmed the TADF process over the triplet-triplet annihilation (Figure 2d). $.122,18$

Density-functional theory (DFT) and time-dependent DFT (TDDFT) calculations at the B3LYP/6-31G(d,p) level revealed the ground state geometry and the excited-state 

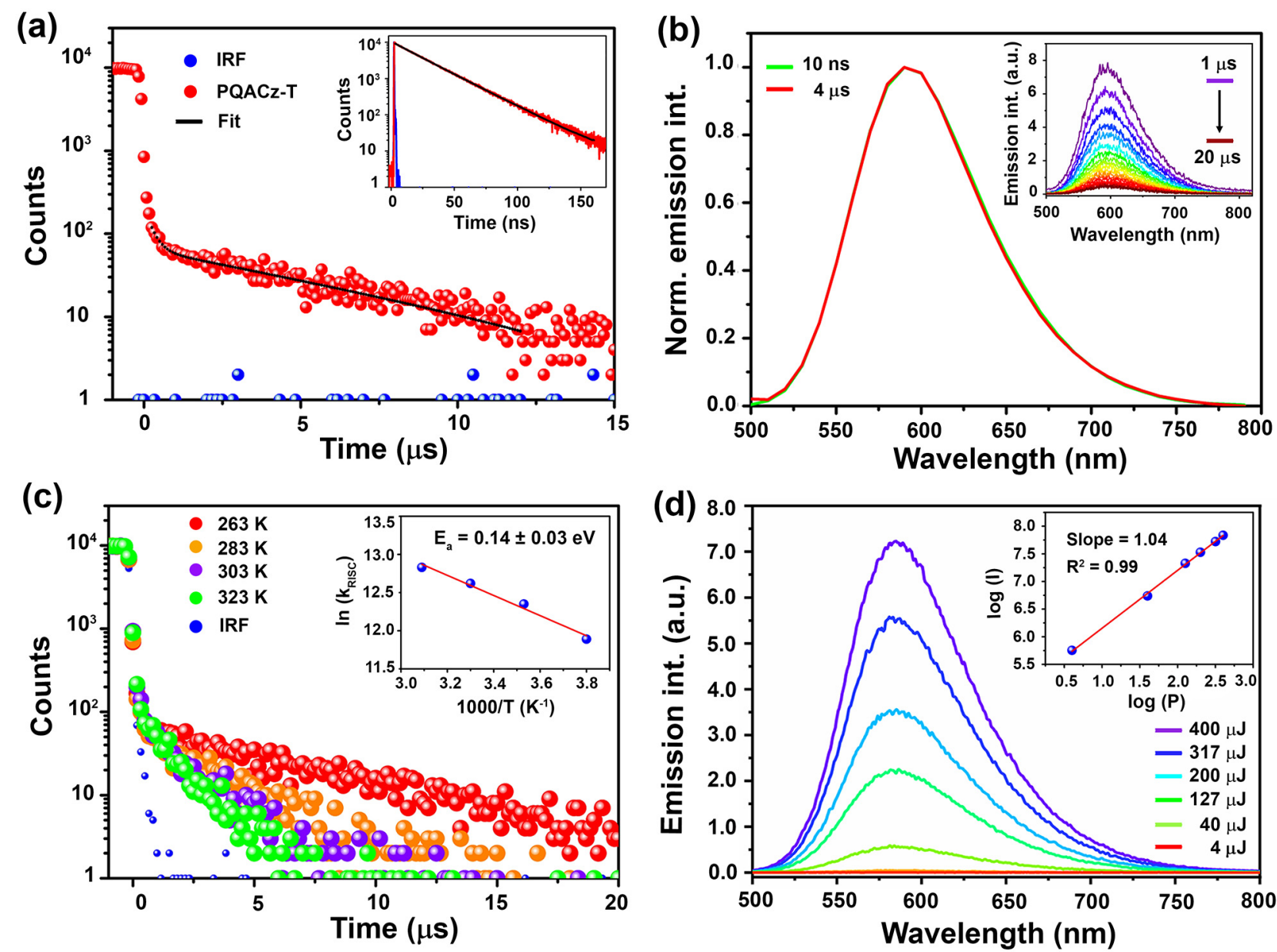

Figure 2 (a) Transient photoluminescence (PL) decay of PQACz-T in deoxygenated toluene at microsecond timescale at room temperature; inset: transient PL decay of PQACZ-T in deoxygenated toluene at the nanosecond timescale at room temperature. (b) Time-resolved emission spectra (TRES) of PQACz-T in deoxygenated toluene at room temperature at microsecond (time delay: $4 \mu \mathrm{s}$, red) and nanosecond timescale (time delay: $10 \mathrm{~ns}$, green), ascertaining delayed fluorescence. The inset figure depicts the TRES analysis of PQACZ-T in deoxygenated toluene recorded with an intensified chargecoupled device (iCCD) camera with a tunable excitation source using optical parametric oscillator (OPO) crystal. (c) Temperature-dependent transient decay spectra of PQACZ-T in deoxygenated toluene; inset: the Arrhenius plot of activation energy ( $E_{\mathrm{a}}$ ) for the reverse intersystem crossing (RISC). (d) Laser power-dependent emission spectra of PQACz-T in deoxygenated toluene under ambient conditions; inset: linear fitting of the emission intensity of PQACz-T as a function of excitation power. $\lambda_{\mathrm{ex}}=470 \mathrm{~nm}$, conc: $10 \mu \mathrm{M}$.

electronic distributions of the molecule, respectively. The dihedral angle between the donor (DTCz) and acceptor (DBPQ) units was found to be $\sim 50^{\circ}$ in the ground-state optimized structure (SI, Figure S10). As shown in Figure 3a, the LUMO $(-2.77 \mathrm{eV})$ was predominantly localized on the central acceptor core, while the HOMO $(-5.17 \mathrm{eV})$ was located on both the donor units. The computed energy gap was found to be $\sim 2.4 \mathrm{eV}$, corroborated well to the optical band gap ( $2.35 \mathrm{eV}$, SI, Figure S2). The TDDFT calculations suggested a low $\Delta E_{\text {ST }}$ value of $0.18 \mathrm{eV}$, similar to that obtained from the experimental results, indicating the possible facile intersystem crossing (ISC)/RISC pathways (Figure 3b, SI, Table S7).

The cyclic voltammogram of PQACz-T indicated one quasi-reversible oxidation reaction and two quasi-reversible reduction reactions (Figure $3 \mathrm{c}$ ). The presence of $t$-butyl groups at the 3,6-positions of the donor unit (DTCz) restricted the oxidative coupling reaction upon electro-oxidation, indicating the electrochemical stability of PQACz-T. ${ }^{44}$ The half-wave oxidation and reduction potentials $\left(E_{1 / 2}\right)$ were used to calculate the HOMO and LUMO energy levels following the equations (1) and (2). ${ }^{45}$ 


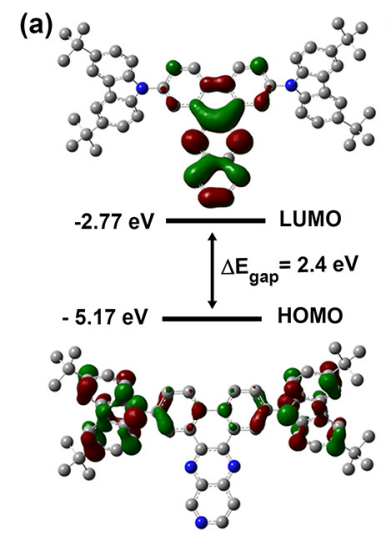

(b)

\begin{tabular}{|l|ccc|}
\multicolumn{4}{c}{ Energy states (eV) } \\
\hline Entry & $\mathrm{S}_{1}$ & $\mathrm{~T}_{1}$ & $\Delta \mathrm{E}_{\mathrm{ST}}$ \\
\hline Theo. & 2.03 & 1.85 & 0.18 \\
\hline Exp. & 2.44 & 2.24 & 0.20 \\
\hline
\end{tabular}

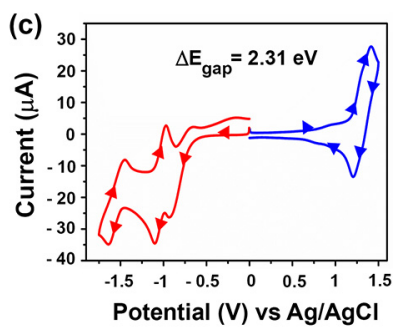

Figure 3 (a) The optimized frontier molecular orbitals (HOMO and LUMO) geometry and the respective energy levels of PQACZ-T calculated through density-functional theory (DFT) at the B3LYP/6-31G (d,p) level. (b) Table representing the lowest energy excited singlet $\left(\mathrm{S}_{1}\right)$, triplet $\left(\mathrm{T}_{1}\right)$ states, and the energy gap ( $\Delta E_{\mathrm{ST}}$ ) obtained from the theoretical (Theo.) calculation using time-dependent density functional theory (TDDFT) and experimental (Exp.) measurements at $77 \mathrm{~K}$. (c) Cyclic voltammogram (scan rate: $100 \mathrm{mV} / \mathrm{s}$ ) of $1 \mathrm{mM}$ solution of PQACz-T in dry dichloromethane using $0.1 \mathrm{M}$ tetrabutylammonium hexafluorophosphate (TBAP) as the supporting electrolyte and glassy carbon as the working electrode. The anodic and cathodic scans are shown in blue and red, respectively. The arrows indicate the direction of the initial scan.

$E_{\text {HOMO }}=-E_{\frac{1}{2}(\mathrm{ox})}-4.8+E_{\frac{1}{2}\left(\mathrm{Fc} / \mathrm{FC}^{+}\right)}$

$E_{\mathrm{LUMO}}=-E_{\frac{1}{2}(\mathrm{red})}-4.8+E_{\frac{1}{2}\left(\mathrm{Fc} / \mathrm{FC}^{+}\right)}$

The estimated HOMO and LUMO energy levels of PQACZ-T were -5.58 and $-3.27 \mathrm{eV}$, respectively, suggesting an electrochemical bandgap of $2.31 \mathrm{eV}$, which closely resembled the theoretically calculated and experimentally obtained optical band gap (SI, Table S8). Half-wave oxidation potential decreased from PQCz-T to PQACZ-T due to the increased donor strength (SI, Figure S11, Table S8).

We investigated the TADF property of PQACz-T in $10 \mathrm{wt} \%$ poly(methyl methacrylate) (PMMA) film. Also, the solid film of the compound was fabricated by drop-casting the methanol dispersion onto a quartz plate and followed by drying under vacuum. The emission maximum of the drop-casted film $(615 \mathrm{~nm})$ was red-shifted as compared to the PMMAdoped film $(565 \mathrm{~nm}$, SI, Figure S12). The red shift in the emission maximum in the solid film might be due to the larger dipole-dipole interactions between the surrounding molecules than in the doped polymer film. ${ }^{46}$ The transient PL decay analysis of the PMMA film showed an average prompt and long-lived decay time of $28 \mathrm{~ns}$ and $56 \mu$ s, respectively (SI, Figure S12, Table S9). TRES measurements of the PMMA film at room temperature signified that the emission

spectrum obtained at the microsecond timescale was identical with the nanosecond timescale spectrum (SI, Figure S13). Furthermore, the laser power-dependent study signified a linear relationship between emission intensity and laser power, thereby confirming the TADF property of the PQACz-T-embedded PMMA film (SI, Figure S14). The transient decay measurements (average prompt and delayed decay time of $15 \mathrm{~ns}$ and $10 \mu \mathrm{s}$, respectively) and TRES analysis of the drop-casted film showed similar observations ascertaining the delayed fluorescence property (SI, Figure S15). The transient decay measurements and TRES analysis of 10 wt\% PMMA doped film of PQCz-T also revealed the delayed fluorescence emission $\left(\lambda_{\mathrm{em}}=540 \mathrm{~nm}, \tau_{\mathrm{DF}(\mathrm{avg})}=58 \mu \mathrm{s}\right.$, SI, Figures S12, S13, Table S9).

The temperature-dependent emission properties of PQACz-T and PQCz-T doped films in PMMA were investigated. The PL intensity was gradually enhanced with increasing temperature (273-313 K) for both PQACz-T- and PQCZ-T-doped PMMA films (SI, Figures S16, S17). The average decay times of the delayed component decreased for

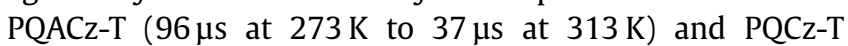
( $104 \mu \mathrm{s}$ at $273 \mathrm{~K}$ to $39 \mu \mathrm{s}$ at $313 \mathrm{~K}$ ) with the gradual increase of temperature (SI, Figures S16, S17, Table S10). The shorter decay time at higher temperatures could be attributed to the facile RISC involving $T_{1} \rightarrow S_{1}$. However, reverse internal conversion from the $\mathrm{T}_{1} \rightarrow \mathrm{T}_{n}$ followed by $\mathrm{T}_{n} \rightarrow \mathrm{S}_{1}$ cannot be ruled out in the doped polymer film. ${ }^{47-49}$

The molecules exhibiting TADF emission in the amorphous aggregated state or in the aqueous dispersions are limited compared to those observed in the solid-state or polymer matrices. ${ }^{25,50,51}$ The effect of aggregation on the emission and decay properties of PQACz-T and PQCz-T was thus checked in tetrahydrofuran (THF)-water binary solvent mixtures with varying water fractions $\left(f_{\mathrm{w}}\right.$, Figure $4, \mathrm{SI}$, Figures S18, S19). The emission spectra $\left(\lambda_{\mathrm{em}}=680 \mathrm{~nm}\right)$ showed a bathochromic shift with a reduced emission intensity compared to that of the THF solution when the water fraction was less than $30 \%$ for PQACz-T (Figure $4 \mathrm{~b}, \mathrm{SI}$, Figure S18). At a lower water fraction $\left(f_{\mathrm{w}} \leq 30 \%\right)$, the enhanced polarity of the solvent mixture might result in a dark TICT state, causing the bathochromic shift with quenched emission as evident from the solvatochromic experiments (Figure 1b, SI, Figure S4). ${ }^{18,37,52}$ The fluorescence intensity thereafter increased, though it was lower than that of the THF solution till $f_{\mathrm{w}} \leq 50 \%$. The plot of the extent of aggregation against the water fractions revealed a steep rise at $70-80 \%$ water fraction, indicating it as the most significant regime of aggregation for PQACZ-T (SI, Figure S20). The maximum emission intensity was observed at $f_{\mathrm{w}}=99 \%$, with $\lambda_{\mathrm{em}}=623 \mathrm{~nm}$, slightly blue-shifted than that of the THF solution $\left(\lambda_{\mathrm{em}}=632 \mathrm{~nm}\right)$. The formation of aggregates impeded the intramolecular motions and nonradiative relaxations, which contributed to a reduction of Stokes shift leading to a blue shift along with a 3-fold rise in the emission intensity of 


\section{(a)}

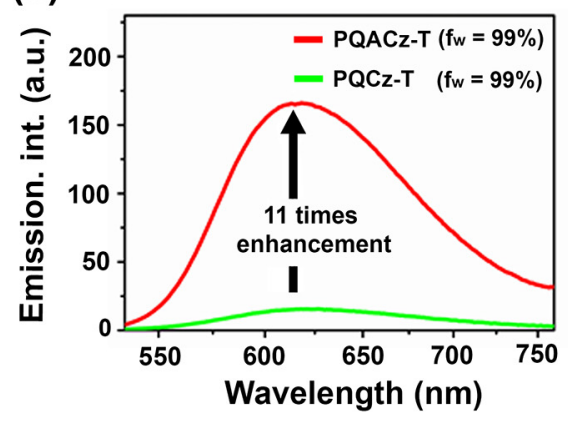

(b)

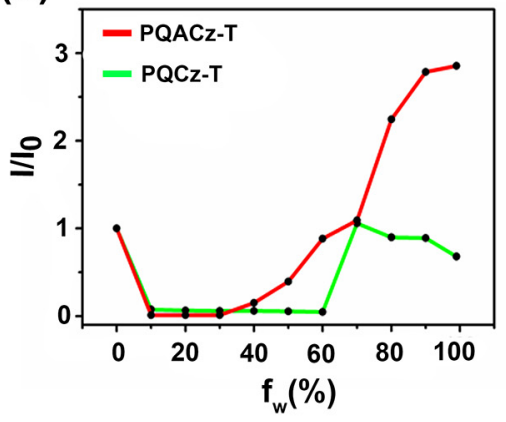

(c)

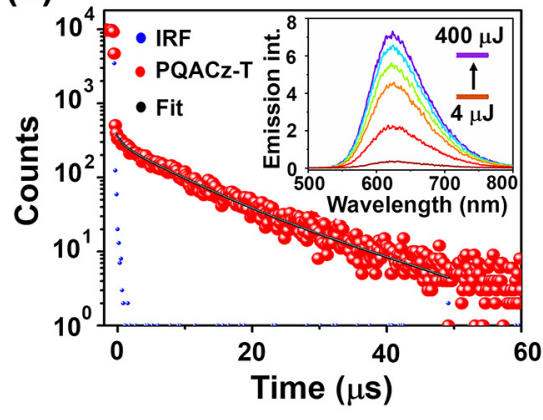

(d)

Increasing water fraction $\left(f_{w}\right)$

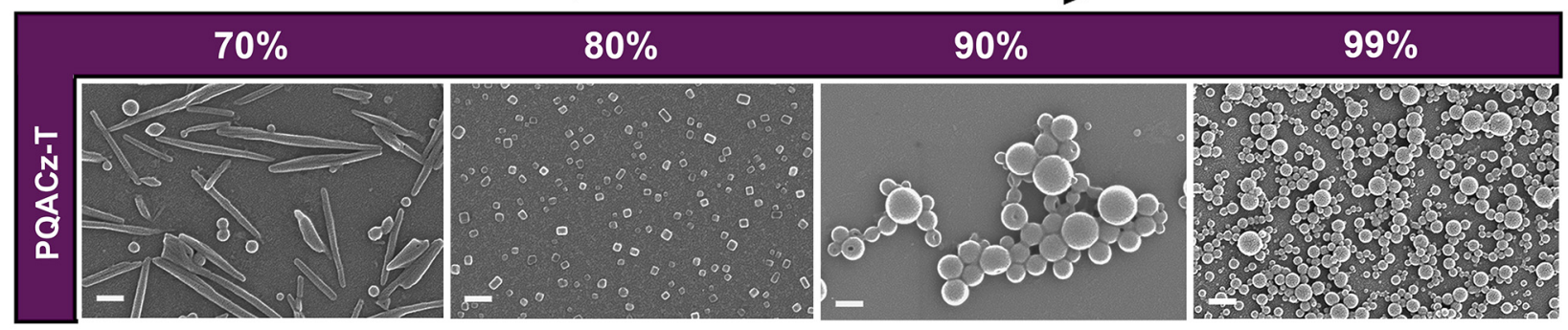

Figure 4 Photophysical and morphological properties of the nanoparticles of PQACZ-T and PQCZ-T formed in THF-water binary solvent mixture: (a) emission spectra of the nanoparticles of PQACZ-T and PQCZ-T in 99\% water fraction at room temperature; (b) intensity ratio versus the fraction of water plot for the nanoparticles of PQACZ-T and PQCZ-T, where $I_{0}$ denotes the intensity at the emission maxima of the pristine molecule in THF, and $I$ denotes the intensity at the emission maxima of different nanoparticles; (c) transient PL decay curves at the microsecond timescale showing the long-lived emission of PQACZ-T nanoparticles formed in $99 \%$ water fraction at room temperature under ambient conditions; the inset depicts the laser powerdependent emission profile of PQACZ-T nanoaggregates $\left(f_{w}=99 \%\right.$ ); (d) field emission scanning electron microscopy (FESEM) images of the nanoparticles of PQACZ-T formed in different water fractions; scale $=200 \mathrm{~nm}$.

the nanoaggregates (99\% water) compared to that of the THF solution (Figure $4 \mathrm{~b})^{52}$

The emission properties of PQCz-T were also monitored under similar conditions. However, no significant enhancement in the fluorescence intensity was observed for the aggregates (Figure 4b, SI, Figure S19). The plot of the extent of aggregation indicated a steep rise in between $60 \%$ and $70 \%$ water fraction, suggesting it as the most prominent aggregation regime (SI, Figure S20). However, a steady decrease in fluorescence intensity was observed for PQCz-T aggregates beyond $70 \%$ water fractions (Figure $4 \mathrm{~b}$ ). The strong $\pi-\pi$ interactions might reduce the emission of PQCz-T in the aggregated state due to nonradiative energy transfer. ${ }^{34,35}$ In contrast, the presence of bulky $t$-butyl groups in PQACz-T could circumvent the $\pi-\pi$ interactions in the aggregated state, leading to fluorescence enhancement. ${ }^{34,35}$

Transient decay measurements were performed with the aggregates at nano- and microsecond timescales (Figure 4c, SI, Figures S21-23, Tables S11-13). Interestingly, the aggregates of PQACZ-T formed at or above $50 \%$ THF-water solvent mixtures showed a microsecond decay ( $\tau_{\mathrm{avg}}=9 \mu \mathrm{s}, f_{\mathrm{w}}=99 \%$ ) along with a prompt nanosecond decay $\left(\tau_{\text {avg }}=29 \mathrm{~ns}\right.$, $f_{\mathrm{w}}=99 \%$ ). Furthermore, the TRES measurements, along with the laser power-dependent study of the nanoaggregates $\left(f_{\mathrm{w}}=99 \%\right)$, confirmed the TADF property (Figure $4 \mathrm{c}, \mathrm{SI}$, Figures S24, S25). The prominent delayed fluorescence emission $\left(\tau_{\text {avg }}=7 \mu \mathrm{s}\right)$ for PQCz-T aggregates was observed only in $f_{\mathrm{w}}=99 \%$ water fraction (SI, Figures S23, S26).

Previous reports indicated the enhanced rate of RISC leading to delayed fluorescence in molecular aggregates..$^{53}$ We observed a gradual increase in the rate of RISC from $3.2 \times 10^{5}$ to $16.7 \times 10^{5} \mathrm{~s}^{-1}$ for PQACZ-T aggregates with increasing water fraction from $50 \%$ to $99 \%$, respectively (Figure 5, SI, Table S14). The enhanced rate of RISC could be attributed to the lowering of $\Delta E_{\mathrm{ST}}$ in the nanoaggregates at higher water fractions facilitating the delayed fluorescence (SI, Figure S27, Table S15). Similar results of low $\Delta E_{S T}$ values in the nanoaggregates of difluoroboron $\beta$-diketonate-based nanoparticles were demonstrated by Yang and co-workers. ${ }^{54}$ The perturbation of the electronic states due to the intermolecular interactions in nanoaggregates results in the reduction of $\Delta E_{\mathrm{ST}} .{ }^{53,54}$ The rate of RISC in PQCZ-T nanoaggregates at $99 \%$ water fraction was found to be $5.5 \times 10^{5} \mathrm{~s}^{-1}$ (SI, Table S14). 


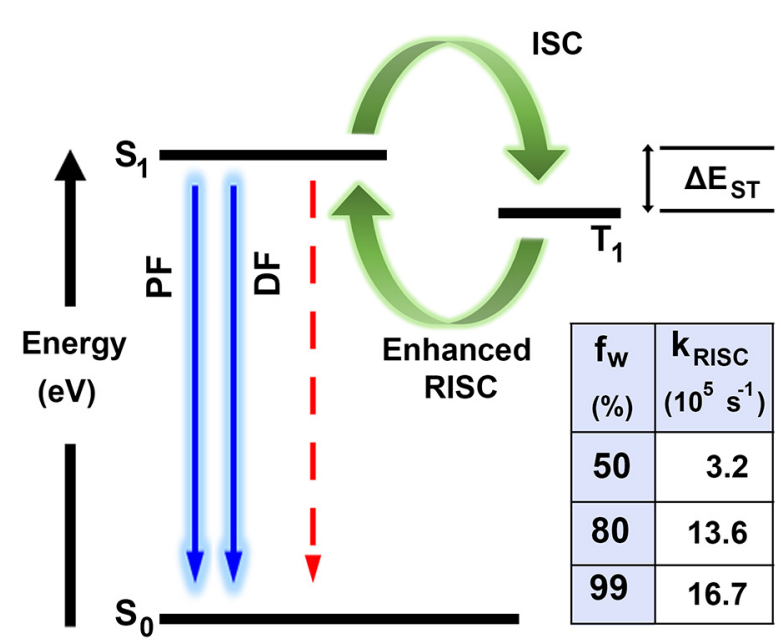

Figure 5 Representative TADF mechanism in PQACz-T nanoparticles. The prompt (PF) and delayed fluorescence (DF) were enhanced with increased water fraction due to the reduction of nonradiative (red) pathways as well as enhancement in intersystem and reverse intersystem crossing (ISC/RISC) rates. The table indicates the enhancement in the rate of RISC values with increasing water fraction ( $f w$ ).

The self-assembly of PQACz-T and PQCz-T towards nanoaggregate formation was probed through field-emission scanning electron microscopy (FESEM) of the drop-casted dispersions. FESEM images of PQACZ-T indicated distinct morphologies of the nanoaggregates formed in different water fractions (Figure 4d). The rod-shaped particles were observed at $f_{\mathrm{W}}=70 \%$, and more spherical particles were formed with increasing water fractions. The average diameter of the spherical nanoaggregates formed at $99 \%$ water fraction was found to be $110 \pm 30 \mathrm{~nm}$. In contrast, only block-shaped particles were formed for PQCz-T under similar conditions (SI, Figure S28). The bulky alkyl chains present in PQACz-T favoured hydrophobic interactions in an aqueous medium resulting in the diverse-shaped aggregate formation. ${ }^{55,56}$

The aqueous dispersion of strongly fluorescent organic nanoparticles (diameter typically $<100 \mathrm{~nm}$ ) has been explored as potential imaging agents. ${ }^{25,49,57}$ However, as mentioned earlier, PQACz-T nanoaggregates fabricated at different compositions of THF-water mixture revealed a broad distribution of particles of diverse sizes and shapes. The molecular self-assembly can be tuned employing amphiphilic surfactants or polymeric micelles through hydrophobic-hydrophobic interactions. ${ }^{58,59}$ Herein, we used Pluronic F-127, a neutral triblock copolymer, to encapsulate PQACz-T in water (Figure 6a). The polymer-encapsulated nanoparticles were prepared from the chloroform solution of PQACz-T and $\mathrm{F}-127$ through slow evaporation of the organic solvent followed by dispersing into water. ${ }^{60}$ The transmission elec- tron microscopy (TEM) images revealed the spherical morphology of F-127-encapsulated PQACZ-T nanoparticles (abbreviated as PQNPs, Figure 6b). The average diameter of PQNPs was found to be much smaller $(20 \pm 4 \mathrm{~nm})$ with a nearly homogeneous particle size distribution as compared to that of PQACz-T nanoaggregates $(110 \pm 30 \mathrm{~nm}$ for $f_{\mathrm{w}}=99 \%$; Figure $6 \mathrm{~b}$, SI, Figure S29).

The aqueous dispersion of PQNPs could be used for noninvasive cellular imaging. The particle size and distribution of PQNPs in aqueous dispersion rather than the solventevaporated state as obtained through TEM would be more relevant for imaging. Hence, to obtain the size of the nanoparticles in the dispersion state, the fluorescence correlation spectroscopy (FCS) analysis was carried out for PQNPs and compared with PQACZ-T nanoaggregates $\left(f_{\mathrm{w}}=99 \%\right.$; Figure 6c). A three-dimensional (3D) diffusion model was used to fit the raw FCS traces; (Eqn. 3) $)^{57,61}$ :

$G_{3 \mathrm{D}}(\tau)=\frac{1}{N} \frac{1}{\left(1+\frac{\tau}{\tau_{\mathrm{D}}}\right)} \frac{1}{\sqrt{1+\frac{\tau}{\omega^{2} \tau_{\mathrm{D}}}}}$

$G_{3 \mathrm{D}}(\tau)$ and $N$ are the autocorrelation function and the average number of particles present in the confocal detection volume, respectively. The structural parameter of the 3D Gaussian confocal volume, $\omega$, is defined through its longitudinal $\left(\omega_{z}\right)$ and transverse radius $\left(\omega_{x y}\right)$, as $\omega=\omega_{z} / \omega_{x y}$. The diffusion time $\left(\tau_{\mathrm{D}}\right)$ and diffusion constant $\left(D_{t}\right)$ of PQNPs were found to be $2.5 \mathrm{~ms}$ and $7.3 \mu \mathrm{m}^{2} \cdot \mathrm{s}^{-1}$, respectively (Figure 6c, SI, Figure S30, Table S16). On the other hand, the $D_{t}$ value for PQACz-T nanoaggregates $\left(f_{\mathrm{w}}=99 \%\right)$ was decreased to $3.9 \mu^{2} \cdot \mathrm{s}^{-1}$ (Figure 6c, SI, Figure S30, Table S16). The hydrodynamic radius of the PQNPs and PQACZ-T aggregates in the dispersion state was calculated using the Stokes-Einstein equation (SI, Eqn. S18). The results revealed a much smaller diameter of PQNPs $(58 \pm 2 \mathrm{~nm})$ as compared to that of PQACz-T aggregates $(110 \pm 10 \mathrm{~nm})$ in the aqueous dispersion (SI, Table S16).

The absorption spectrum of PQNPs in water was found to be quite similar to that of PQACz-T in the solution state (Figure 6d). The emission of PQNPs was found to show a peak at $628 \mathrm{~nm}$ with an appreciable quantum yield of $\sim 20 \pm 0.3 \%$. The transient decay measurements and TRES analysis along with laser power-dependent study confirmed the TADF in PQNPs (Figure 6e, f, SI, Figure S31). The average prompt and delayed decay times were found to be $26 \mathrm{~ns}$ and $23 \mu \mathrm{s}$, respectively (Figure 6e, Table S17). The smaller particle size, water dispersibility, longer emission wavelength, high quantum yield, and excellent photostability prompted us to check the subcellular internalization of PQNPs (SI, Figure S32).

In order to evaluate the cytocompatibility, MTT assays were carried out using HeLa cells. PQNPs showed low toxicity despite incubating the cells with high concentrations of up to $150 \mu \mathrm{g} / \mathrm{mL}$ (SI, Figure S33). Hence, HeLa cells were in- 
(a)
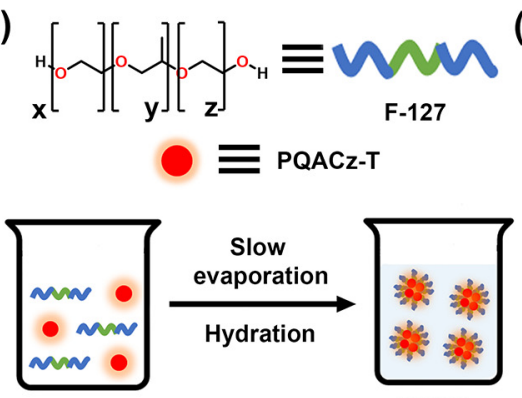

PQNPS (b)

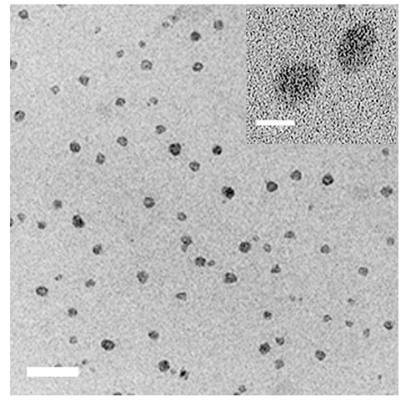

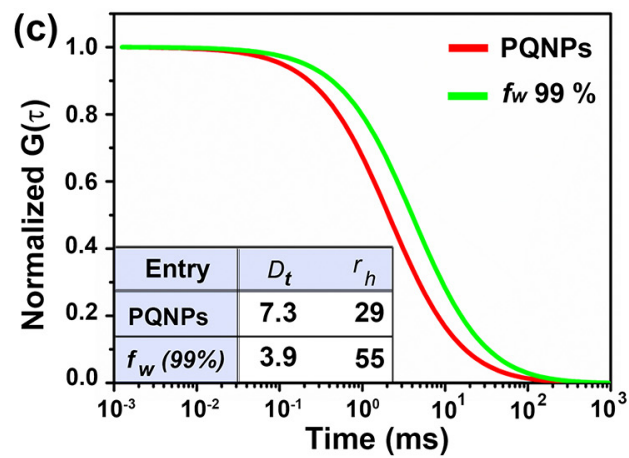

(d)

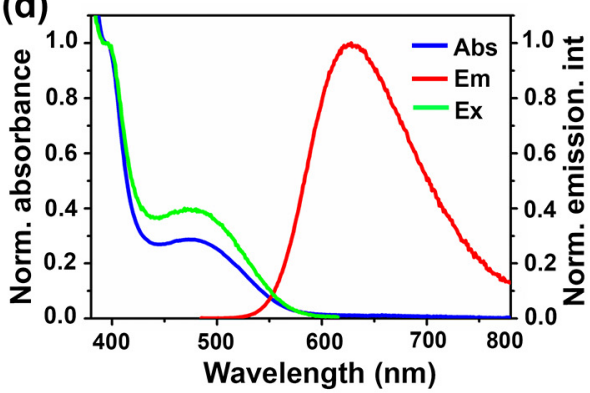

(e)

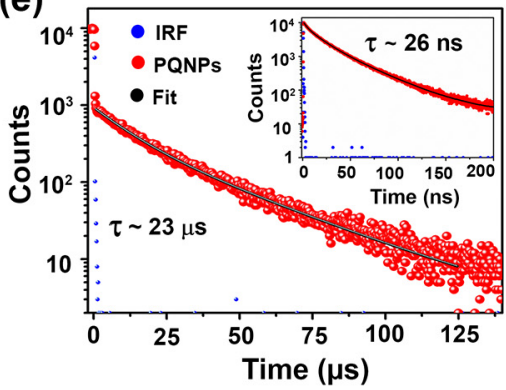

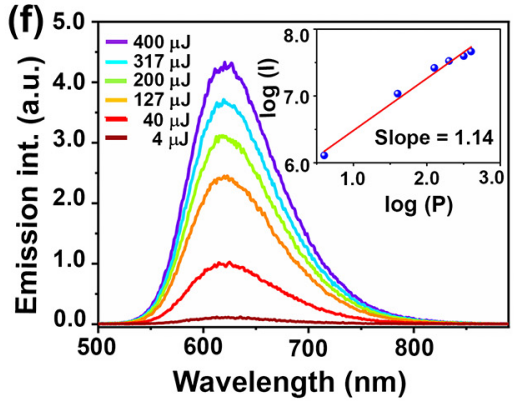

Figure 6 (a) Schematic representation depicting the preparation of aqueous dispersion of PQNP nanoprobe employing Pluronic F-127 triblock copolymer. (b) Transmission electron microscopy (TEM) images of PQNPs (scale $=100 \mathrm{~nm}$ ); inset: zoomed view of PQNPs (scale $=20 \mathrm{~nm})$. (c) Normalized fluorescence correlation spectroscopy (FCS) traces $\left(\lambda_{\mathrm{ex}}=470 \mathrm{~nm}, \lambda_{\mathrm{em}}=532-800 \mathrm{~nm}\right)$ of PQACz-T $(5 \mathrm{nM})$ in the form of PQNPs (red) and nanoparticles $\left(f_{\mathrm{w}}=99 \%\right.$, green); the FCS data are fitted using Eqn. (3); inset: diffusion coefficients $\left(D_{t}, \mu \mathrm{m}^{2} \cdot \mathrm{s}^{-1}\right)$ and hydrodynamic radius $\left(r_{\mathrm{h}}, \mathrm{nm}\right)$ of PQNPs and nanoaggregates $\left(f_{\mathrm{w}}=99 \%\right.$ ), respectively. (d) Normalized absorption, emission, and excitation spectra of PQNPs in aqueous dispersion. (e) Transient PL decay curves at the microsecond timescale of PQNPs at room temperature under ambient conditions; inset: transient PL decay curves at the nanosecond timescale of PQNPs. (f) Laser power-dependent emission spectra of PQNPs under ambient conditions; inset: linear fitting of the emission intensity of the PQNPs as a function of excitation power.

cubated with the aqueous dispersion of PQNPs $(125 \mu \mathrm{g} / \mathrm{mL})$ for 15 minutes. A distinct punctate pattern was noticeable in the confocal laser scanning microscopy images, indicating the internalization of PQNPs in the LDs (SI, Figure S34). The intracellular localization of PQNPs was confirmed in live He-
La cells by the co-staining experiment with BODIPY 493/503 (commercial dye for staining of LDs, Figure 7a-c). The corresponding Pearson's coefficient of colocalization value of 0.96 indicated the LD specificity of the probe (Figure 7d).
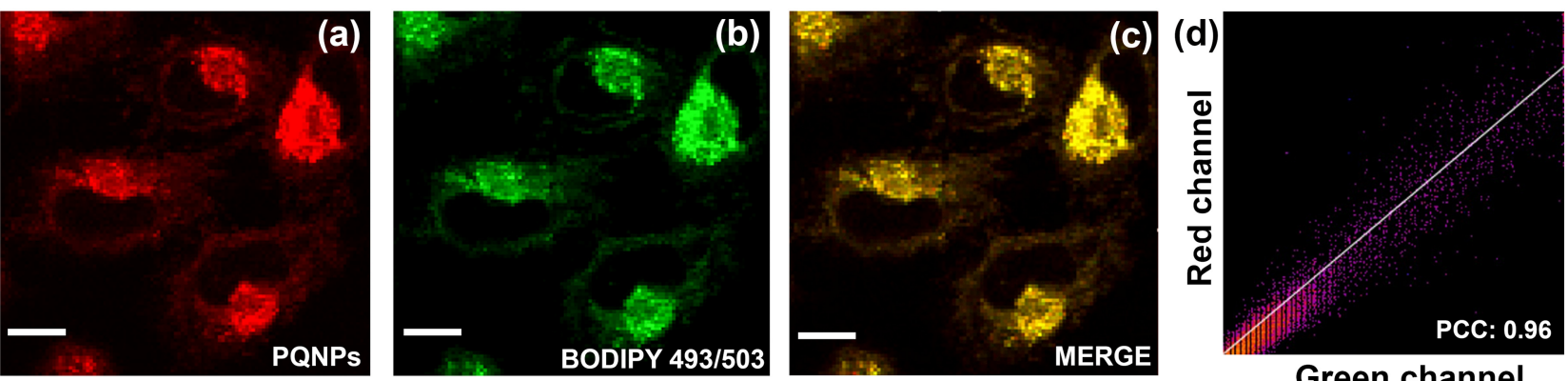

Green channel

Figure 7 Confocal laser scanning microscopy (CLSM) colocalization images of HeLa cells incubated with (a) PQNPs $\left(\lambda_{\mathrm{ex}}=470 \mathrm{~nm}, \lambda_{\mathrm{em}}=610-800 \mathrm{~nm}\right)$, (b) lipid droplet tracker dye, BODIPY $493 / 503\left(\lambda_{\mathrm{ex}}=470 \mathrm{~nm}, \lambda_{\mathrm{em}}=510-530 \mathrm{~nm}\right)$, and (c) merged image. (d) Pearson's colocalization coefficient of red (PQNPs) and green (BODIPY 493/503) channels was found to be 0.96 , indicating the lipid droplet staining by PQNPs. Scale $(a-c)=20 \mu m$. 
LDs are universal lipid-rich storage organelles consisting of hydrophobic neutral lipids with an outer phospholipid monolayer. ${ }^{62,63}$ The inherent hydrophobic nature of PQACZ-T (calculated $\log P=19.4$ ) was attributed to the LD specificity of PQNPs, which was unaffected by the influence of F-127 micelles (SI, Table S18) ${ }^{64,65}$ Further, the colocalization experiments with MitoTracker Green and LysoTracker Green confirmed no specific staining of mitochondria and lysosome, respectively, by PQNPs (SI, Figure S35). PQNPs have longer fluorescence lifetimes in the order of microseconds than the short-lived autofluorescence or background fluorescence in the cellular environments ( $<10 \mathrm{~ns})$. The long-lived emissive characteristic in the aqueous medium suggests PQNPs as promising candidates for future applications in time-gated imaging.

\section{Conclusions}

In summary, we rationalized the variation of the excitedstate properties due to the substitution of a $t$-butyl group in the donor carbazole moiety in multichromophoric D-A-D compounds, PQACz-T (with alkyl substitution) and PQCz-T (without alkyl substitution), having a rigid dibenzopyridoquinoxaline acceptor core. Both compounds exhibited TADF in the doped polymer films. PQACz-T was found to be self-assembled to molecular nanoaggregates of diverse size and shape in THF-water mixtures, presumably due to the hydrophobic interactions imparted by the $t$-butyl units. PQACZ-T nanoaggregates exhibited bright red emission along with delayed fluorescence in the aqueous dispersion. Further, the smaller-sized, homogeneous distribution of fluorescent nanoparticles in water was fabricated upon encapsulating PQACz-T in a triblock copolymer, F-127 matrix, for intracellular imaging. Cytocompatible, polymer-encapsulated PQACZ-T nanoparticles were employed for specific imaging of LDs in HeLa cells. The present study paves the way for further exploration of the molecular self-assembly processes of TADF nanoaggregates for specific intracellular sensing and imaging.

\section{Experimental Section}

Synthesis of 2,7-bis(3,6-di-tert-butyl-9H-carbazol-9-yl)dibenzo[f,h]pyrido[3,4-b]quinoxaline (PQACz-T): To a mixture of P3 (100 mg, 1 equiv.), 3,6 di-t-butylcarbazole (DTCz, 2.1 equiv.), $\mathrm{Pd}_{2}$ (dba) $)_{3}$ ( 0.15 equiv.), $t$-BuOK ( 4 equiv.) and $\mathrm{X}$-Phos ( 0.15 equiv.), $10 \mathrm{~mL}$ of toluene was added and stirred under an $\mathrm{Ar}$ atmosphere at $110^{\circ} \mathrm{C}$ for $24 \mathrm{~h}$. After cooling the reaction mixture to room temperature, the reaction was quenched by the addition of $10 \%$ aqueous $\mathrm{NaHCO}_{3}$ and $20 \mathrm{~mL}$ distilled water. The organic layer was then extracted with dichloromethane $(3 \times 15 \mathrm{~mL})$, dried over anhydrous
$\mathrm{MgSO}_{4}$, and concentrated under vacuum. The product was purified by column chromatography (neutral alumina, $10 \% \mathrm{DCM} /$ hexane) to afford an orange solid with a yield of $65 \%$ (123 mg, Scheme S4, mp: $340^{\circ} \mathrm{C}$, based on the decomposition colour change).

${ }^{1} \mathrm{H}$ NMR (700 MHz, $\left.\mathrm{CDCl}_{3}\right): \delta 9.76(\mathrm{~s}, 1 \mathrm{H}), 9.69(\mathrm{~m}, 2 \mathrm{H})$, $8.87(\mathrm{~m}, 1 \mathrm{H}), 8.81(\mathrm{~m}, 2 \mathrm{H}), 8.23(\mathrm{~s}, 4 \mathrm{H}), 8.11(\mathrm{~m}, 3 \mathrm{H}), 7.56$ $(\mathrm{m}, 8 \mathrm{H}), 1.52(\mathrm{~s}, 36 \mathrm{H}) .{ }^{13} \mathrm{C} \mathrm{NMR}\left(126 \mathrm{MHz}, \mathrm{CDCl}_{3}\right): \delta 155.44$, $146.67,145.83,144.19,143.70,143.46,139.13,138.50$, $137.18,131.23,129.79,124.99,124.58,124.07,123.83$, $121.40,116.53,109.25,34.85,32.06$. HRMS (APCI): calculated for $\mathrm{C}_{59} \mathrm{H}_{57} \mathrm{~N}_{5} \quad[\mathrm{M}]^{+}: \quad 836.1400 \mathrm{~g} \cdot \mathrm{mol}^{-1}$, found: $836.4711 \mathrm{~g} \cdot \mathrm{mol}^{-1}$.

The detailed synthetic procedures and yields of the intermediate compounds are mentioned in the SI. The thin films were fabricated by spin-coating the toluene solution of $10 \mathrm{wt} \%$ of PQACz-T and PQCz-T in PMMA, followed by drying under vacuum. For the fabrication of nanoaggregates of the compounds, a stock solution of $1 \mathrm{mM}$ was prepared in dry THF. $30 \mu \mathrm{L}$ of the stock solution was rapidly added to the binary solvent mixture of THF-water (total volume: $3 \mathrm{~mL}$ ) under sonication for $1 \mathrm{~min}$. Polymer-encapsulated nanoparticles (PQNPs) for intracellular imaging were fabricated using a stock solution of PQACz-T (1 mg) and polyethylenepolypropylene glycol (Pluronic F-127, $12 \mathrm{mg}$ ) in $\mathrm{CHCl}_{3}$ $(1.1 \mathrm{~mL})$. Subsequently, the evaporation of organic solvent resulted in a film, which was dispersed in distilled water. The aqueous dispersion upon filtration through a nanoporous membrane $(0.2 \mu \mathrm{m})$ was used for further experiments.

\section{Funding Information}

DST-SERB (Project no. EMR/2017/000233), Council of Scientific and Industrial Research (CSIR), New Delhi (No.01 (2878)/17/EMR-II).

\section{Acknowledgment}

We thank the infrastructural support from Indian Institute of Science Education and Research Bhopal (IISERB), and the TEM facility at the Department of Chemistry, IISERB, supported through the DST FIST programme. S.D. and B.S. thank IISERB, and S.K. and M.S. thank University Grants Commission and Council of Scientific and Industrial Research for a fellowship, respectively. S.D. acknowledges Ms. Nitu Saha for help with the MTT assay and Ms. Ashima Negi and Dr. Ankur Gupta at IISERB for helping in the laser fluence-dependent study. 


\section{Supporting Information}

Details of synthesis, characterization, absorption, emission and lifetime data of PQACz-T, time-resolved emission spectra, TADF characteristics, and self-assembly behaviour of $\mathrm{PQACz}-\mathrm{T}$ and $\mathrm{PQCz}-\mathrm{T}$, supporting colocalization experiments with PQACz-T nanoparticles and the comparative analysis of lipid droplet imaging are given in the Supporting Information.

Supporting Information for this article is available online at https://doi.org/10.1055/a-1679-9558.

\section{Conflict of Interest}

The authors declare the following competing financial interest. A patent application has been filed on "Dibenzopyridoquinoxaline based derivatives" with A. Patra, B. Sk, M. Sarkar and S. Kundu as inventors. ${ }^{66}$

\section{References}

(1) Uoyama, H.; Goushi, K.; Shizu, K.; Nomura, H.; Adachi, C. Nature 2012, 492, 234.

(2) Tao, Y.; Yuan, K.; Chen, T.; Xu, P.; Li, H.; Chen, R.; Zheng, C.; Zhang, L.; Huang, W. Adv. Mater. 2014, 26, 7931.

(3) Liu, Y.; Li, C.; Ren, Z.; Yan, S.; Bryce, M. R. Nat. Rev. Mater. 2018, 3 , 18020.

(4) Im, Y.; Kim, M.; Cho, Y. J.; Seo, J. A.; Yook, K. S.; Lee, J. Y. Chem. Mater. 2017, 29, 1946.

(5) Yang, Z.; Mao, Z.; Xie, Z.; Zhang, Y.; Liu, S.; Zhao, J.; Xu, J.; Chi, Z.; Aldred, M. P. Chem. Soc. Rev. 2017, 46, 915.

(6) Chen, X. K.; Kim, D.; Brédas, J. L. Acc. Chem. Res. 2018, 51, 2215.

(7) Pashazadeh, R.; Pander, P.; Bucinskas, A.; Skabara, P. J.; Dias, F. B.; Grazulevicius, J. V. Chem. Commun. 2018, 54, 13857.

(8) Zhu, Z.; Tian, D.; Gao, P.; Wang, K.; Li, Y.; Shu, X.; Zhu, J.; Zhao, Q. J. Am. Chem. Soc. 2018, 140, 17484.

(9) Paisley, N. R.; Halldorson, S. V.; Tran, M. V.; Gupta, R.; Kamal S.; Algar, R.; Hudson, Z. M. Angew. Chem. Int. Ed. 2021, 60, 18630.

(10) Kawasumi, K.; Wu, T.; Zhu, T.; Chae, H. S.; Voorhis, T. V.; Baldo, M. A.; Swager, T. M. J. Am. Chem. Soc. 2015, 137, 11908.

(11) Data, P.; Pander, P.; Okazaki, M.; Takeda, Y.; Minakata, S.; Monkman, A. P. Angew. Chem. Int. Ed. 2016, 55, 5739.

(12) Kuila, S.; Garain, S.; Banappanavar, G.; Garain, B. C.; Kabra, D.; Pati, S. K.; George, S. J. Phys. Chem. B 2021, 125, 4520.

(13) Imagawa, T.; Hirata, S.; Totani, K.; Watanabe, T.; Vacha, M. Chem. Commun. 2015, 51, 13268.

(14) Ward, J. S.; Nobuyasu, R. S.; Batsanov, A. S.; Data, P.; Monkman, A. P.; Dias, F. B.; Bryce, M. R. Chem. Commun. 2016, 52, 2612.

(15) Klimash, A.; Pander, P.; Klooster, W. T.; Coles, S. J.; Data, P.; Dias, F. B.; Skabara, P. J. J. Mater. Chem. C 2018, 6, 10557.

(16) Santos, P. L.; Chen, D.; Rajamalli, P.; Matulaitis, T.; Cordes, D. B.; Slawin, A. M. Z.; Jacquemin, D.; Colman, E. Z.; Samuel, I. D. W. ACS Appl. Mater. Interfaces 2019, 11, 45171.

(17) Xie, F. M.; Li, H. Z.; Dai, G. L.; Li, Y. Q.; Cheng, T.; Xie, M.; Tang, J. X.; Zhao, X. ACS Appl. Mater. Interfaces 2019, 11, 26144.

(18) Sk, B.; Sharma, S.; James, A.; Kundu, S.; Patra, A. J. Mater. Chem. C 2020, 8, 12943.
(19) Voll, C. C. A.; Markopoulos, G.; Wu, T. C.; Welborn, M.; Engelhart, J. U.; Rochat, S.; Han, G. G. D.; Sazama, G. T.; Lin, T. A.; Voorhis, T. V.; Baldo, M. A.; Swager, T. M. Org. Mater. 2020, 2, 1.

(20) Xiao, Y. F.; Chen, J. X.; Li, S; Tao, W. W.; Tian, S.; Wang, K.; Cui, X.; Huang, Z.; Zhang, X. H.; Lee, C. S. Chem. Sci. 2020, 11, 888.

(21) Wang, S.; Cheng, Z.; Song, X.; Yan, X.; Ye, K.; Liu, Y.; Yang, G.; Wang, Y. ACS Appl. Mater. Interfaces 2017, 9, 9892.

(22) Wei, W.; Yang, Z.; Chen, X.; Liu, T.; Mao, Z.; Zhao, J.; Chi, Z. J. Mater. Chem. C 2020, 8, 3663.

(23) Bhattacharjee, I.; Acharya, N.; Bhatia, H.; Ray, D. J. Phys. Chem. Lett. 2018, 9, 2733.

(24) Li, C.; Duan, R.; Liang, B.; Han, G.; Wang, S.; Ye, K.; Liu, Y.; Yi, Y.; Wang, Y. Angew. Chem. Int. Ed. 2017, 56, 11525.

(25) Hu, W.; Guo, L.; Bai, L.; Miao, X.; Ni, Y.; Wang, Q.; Zhao, H.; Xie, M.; Li, L.; Lu, X.; Huang, W.; Fan, Q. Adv. Healthcare Mater. 2018, 7, 1800299 .

(26) Zhang, Y. L.; Ran, Q.; Wang, Q.; Liu, Y.; Hänish, C.; Reineke, S.; Fan, J.; Liao, L. S. Adv. Mater. 2019, 31, 1902368

(27) Ni, F.; Zhu, Z.; Tong, X.; Zeng, W.; An, K.; Wei, D.; Gong, S.; Zhao, Q.; Zhou, X.; Yang, C. Adv. Sci. 2019, 6, 1801729.

(28) Jena, S.; Dhanalakshmi, P.; Bano, G.; Thilagar, P. J. Phys. Chem. B 2020, 124, 5393.

(29) Englman, R.; Jortner, J. Mol. Phys. 1970, 18, 145.

(30) Srujana, P.; Sudhakar, P.; Radhakrishnan, T. P. J. Mater. Chem. C 2018, 6, 9314

(31) Chen, Y.; Lam, J. W. Y.; Kwok, R. T. K.; Liu, B.; Tang, B. Z. Mater. Horiz. 2019, 6, 428.

(32) Kim, J. H.; Yun, J. H.; Lee, J. Y. Adv. Opt. Mater. 2018, 6, 1800255.

(33) Kuila, S.; Ghorai, A.; Samanta, P. K.; Siram, R. B. K.; Pati, S. K.; Narayan, K. S.; George, S. Chem. Eur. J. 2019, 25, 16007.

(34) Lin, M. J.; Jiménez, Á. J.; Burschka, C.; Würthner, F. Chem. Commun. 2012, 48, 12050.

(35) Fan, Y.; Li, Q.; Li, Z. Mater. Chem. Front. 2021, 5, 1525.

(36) Gong, Y.; Zhang, Y.; Yuan, W. Z.; Sun, J. Z.; Zhang, Y.J. Phys. Chem. C 2014, 118, 10998.

(37) Sk, B.; Khodia, S.; Patra, A. Chem. Commun. 2018, 54, 1786.

(38) Zheng, X.; Zhu, W.; Ni, F.; Ai, H.; Gong, S.; Zhou, X.; Sessler, J. L.; Yang, C. Chem. Sci. 2019, 10, 2342.

(39) Singha, S.; Kim, D.; Roy, B.; Sambasivan, S.; Moon, H.; Rao, A. S.; Kim, J. Y.; Joo, T.; Park, J. W.; Rhee, Y, M.; Wang, T.; Kim, K. H.; Shin, Y, H.; Jung, J.; Ahn, K. H. Chem. Sci. 2015, 6, 4335.

(40) Sasaki, S.; Drumen, G. P. C.; Konishi, G. J. Mater. Chem. C 2016, 4, 2731.

(41) Reja, S. I.; Khan, I. A.; Bhalla, V.; Kumar, M. Chem. Commun. 2016, $52,1182$.

(42) Abdel-Shafi, A. A.; Worrall, D. R. J. Photochem. Photobiol., A. 2005, $172,170$.

(43) Cheng, Y. H.; Belyaev, A.; Ho, M. L.; Koshevoy, I. O.; Chou, P. T. Phys. Chem. Chem. Phys. 2020, 22, 27144.

(44) Zhang, D.; Cai, M.; Zhang, Y.; Zhang, D.; Duan, L. Mater. Horiz. 2016, 3, 145.

(45) Pommerehne, J.; Vestweber, H.; Guss, W.; Mahrt, R. F.; Bässler, H.; Porsch, M.; Daub, J. Adv. Mater. 1995, 7, 551.

(46) Kim, H. S.; Park, S. R.; Suh, M. C. J. Phys. Chem. C 2017, 121, 13986.

(47) Lin, J. A.; Li, S. W.; Liu, Z. Y.; Chen, D. G.; Huang, C. Y.; Wei, Y. C.; Chen, Y. Y.; Tsai, Z. H.; Lo, C. Y.; Hung, W. Y.; Wong, K. T.; Chou, P. T. Chem. Mater. 2019, 31, 5981.

(48) Guo, J.; Fan, J.; Lin, L.; Zeng, J.; Liu, H.; Wang, C. K.; Zhao, Z.; Tang, B. Z. Adv. Sci. 2019, 6, 1801629.

(49) Qi, S.; Kim, S.; Nguyen, V. N.; Kim, Y.; Niu, G.; Kim, G.; Kim, S. J.; Park, S.; Yoon, J. ACS Appl. Mater. Interfaces 2020, 12, 51293. 
(50) Tsuchiya, Y.; Ikesue, K.; Nakanotani, H.; Adachi, C. Chem. Commun. 2019, 55, 5215.

(51) Li, X.; Baryshnikov, G.; Ding, L.; Bao, X.; Li, X.; Lu, J.; Liu, M.; Shen, S.; Luo, M.; Zhang, M.; Årgen, H.; Wang, X.; Zhu, L. Angew. Chem. Int. Ed. 2020, 59, 7548.

(52) Qin, W.; Feng, G.; Li, M.; Yang, Z.; Liu, B.; Tang, B. Z. Adv. Funct. Mater. 2014, 24, 635.

(53) Zhang, K.; Zhang, Y.; Ma, Y.; Fan, J.; Wang, C. K.; Lin, L. J. Phys. Chem. A 2020, 124, 8540.

(54) Zhang, H.; Chen, P. Z.; Niu, L. Y.; Yang, Q. Z. Mater. Chem. Front. 2020, 4, 285.

(55) Li, N.; Liu, Y. Y.; Li, Y.; Zhuang, J. B.; Cui, R. R.; Gong, Q.; Zhao, N.; Tang, B. Z. ACS Appl. Mater. Interfaces 2018, 10, 24249.

(56) Kundu, S.; Chowdhury, A.; Nandi, S.; Bhattacharyya, K.; Patra, A. Chem. Sci. 2021, 12, 5874.

(57) Middha, E.; Liu, B. ACS Nano 2020, 14, 9228.

(58) Cheng, H. B.; Li, Y.; Tang, B. Z.; Yoon, J. Chem. Soc. Rev. 2020, 49, 21.

(59) Chen, C.; Wylie, R. A. L.; Klinger, D.; Connal, L. A. Chem. Mater. 2017, 29, 1918.

(60) Qi, J.; Sun, C.; Zebibula, A.; Zhang, H.; Kwok, R. T. K.; Zhao, X.; Xi, W.; Lam, J. W. Y.; Qian, J.; Tang, B. Z. Adv. Mater. 2018, 30, 1706856.

(61) Dong, C.; Irudayaraj, J. J. Phys. Chem. B 2012, 116, 12125.

(62) Martin, S.; Parton, R. G. Nat. Rev. Mol. Cell Biol. 2006, 7, 373.

(63) Sk, B.; Thakre, P. K.; Tomar, R. S.; Patra, A. Chem. Asian J. 2017, 12, 2501.

(64) Rakshit, S.; Das, S.; Govindaraj, V.; Maini, R.; Kumar, A.; Datta, A. J. Phys. Chem. B 2020, 124, 10282.

(65) Guo, X.; Tang, B.; Wu, H.; Wu, Q.; Xie, Z.; Yu, C.; Hao, E.; Jiao, L. Mater. Chem. Front. 2021, 5, 3664.

(66) Patra, A.; Sk, B.; Sarkar, M.; Kundu, S. Indian Pat. Appl. 201921041228, October 11, 2019. 